\title{
MORPHOLOGICAL GRADIENT OPERATORS FOR COLOUR IMAGES
}

\author{
Adrian N. Evans \\ Department of Electronic and Electrical Engineering \\ University of Bath, BA2 7AY, UK \\ Email: A.N.Evans@bath.ac.uk
}

\begin{abstract}
A new morphological gradient operator for colour images is introduced that can be viewed as a direct extension of the well known morphological gradient. In this approach, each pixel is considered as multivariate data and its output is the maximum distance between any two points within a structuring element, determined by a norm. In contrast with other nonlinear schemes, this approach reduces to the morphological gradient for single channel images. It is also computationally efficient and responds well to step edges. To overcome any sensitivity to noise a robust colour morphological gradient operator is proposed that rejects outlying vector pairs before determining the maximum distance. Results show the effectiveness of the techniques.
\end{abstract}

\section{INTRODUCTION}

Determining the gradient of an image is a fundamental image processing operation that is often used as a precursor to other, more advanced operations such as feature extraction and segmentation. Increasingly, image processing techniques have to accommodate multidimensional data, such as that found in colour and multispectral imagery, where the application of greyscale gradient techniques to individual channels is often inadequate. The extension of second order differential methods to multispectral images has been considered by Cumani [1]. Compass edge detectors have been applied to colour images in [2] and more recently by Ruzon and Tomasi [3], who have further considered junction and corner detection [4]. Using Bayesian theory, the maximum a posteriori criterion was used to detect and locate edges in vector images by Djuric and Fwu [5]. In contrast to the above approaches, this paper considers gradient operators for colour images based on mathematical morphology [6].

The classical morphological gradient operator for greyscale images is the difference between a dilation and an erosion [7]. Simple operators of this type are over-sensitive to image noise and some alternatives for practical morphological edge detection were proposed by Lee et al. [8]. Attempts to extend morphological operations to colour images have been hampered by the absence of an explicit ordering for multivariate data and this restriction is equally applicable to the morphological gradient. In [9], lexicographical ordering is used to develop a multi-scale gradient operator for morphological segmentation. An alternative approach is Reduced Ordering (R-ordering), in which the ordering is performed on a set of scalar values derived from the vector data, as used by the vector order statistics-based colour edge detectors of Trahanias and Venetsanopoulos [10, 11]. As this work is the most directly related to that presented here, it is reviewed in section 2. In contrast, the colour morphological gradient operators proposed in this paper do not require an explicit vector ordering, producing the simple and effective operators that are described in section 3. Results and conclusions are given in sections 4 and 5 respectively.

\section{VECTOR ORDER STATISTICS COLOUR EDGE DETECTION}

In the R-ordering employed by [10] and [11], the set of vectors $\mathbf{X}^{1}, \mathbf{X}^{2}, \cdots, \mathbf{X}^{n}$ are ordered according to their scalar distances, $d_{i}$ s, given by

$$
d_{i}=\sum_{k=1}^{n}\left\|\mathbf{X}^{i}-\mathbf{X}^{k}\right\|_{p}
$$

where $p$ is a norm, to give the ordered sequence $\mathbf{X}^{1} \leq$ $\mathbf{X}^{2} \leq \cdots \leq \mathbf{X}^{n}$ in which $\mathbf{X}^{1}$ is the vector median [12]. Following from this ordering, the vector range edge detector (VRED) is defined as

$$
V R E D=\left\|\mathbf{X}^{(n)}-\mathbf{X}^{(1)}\right\|_{p}
$$

and measures the distance from the median to the extremum vector. However, this detector will respond to a single noisy pixel and to improve its robustness the distances to the $k$ highest ranked vectors can be taken into account, resulting in the minimum vector range edge detector (MVRED),

$$
\begin{gathered}
M V R E D=\min _{j}\left\{\left\|\mathbf{X}^{(n-j+1)}-\mathbf{X}^{(1)}\right\|_{p}\right\} \\
j=1,2, \cdots, k ; k<n
\end{gathered}
$$


where $k$ can be estimated by considering the numbers of pixels on the smaller side of an edge. Further details of this and other related vector edge detectors are given in [10] and [11].

\section{COLOUR MORPHOLOGICAL GRADIENT OPERATORS}

The classical definition of the morphological gradient of a greyscale image is [7]

$$
\nabla(f)=\delta_{g}(f)-\epsilon_{g}(f)
$$

for a structuring element $g$. To develop a colour morphological operator, the greyscale gradient of (4) can alternatively be expressed as

$$
\begin{aligned}
\nabla(f) & =\max _{x \in g}\{f(x)\}-\min _{x \in g}\{f(x)\} \\
& =\max (|f(x)-f(y)|) \quad \forall x, y \in g .
\end{aligned}
$$

Hence, the morphological gradient can be viewed as the greatest absolute intensity difference between any two pixels within the structuring element; a definition that can easily be extended to give the colour morphological gradient (CMG) operator. Denoting $\{N\}=1,2, \cdots, N$ as the set of $N$ vectors contained within a structuring element $g$, the $\mathrm{CMG}$ can then be defined as

$$
C M G=\max _{i, j \in\{N\}}\left\{\left\|\mathbf{X}^{(i)}-\mathbf{X}^{(j)}\right\|_{p}\right\}
$$

whose response is the distance between the two vectors in the set that are furthest apart, see figure 1. Compared with the VRED, (6) reduces to the morphological gradient of (4) for single channel images and therefore has the potential to provide a better estimate of the gradient. For example, the single channel form of (2) measures the difference between the median and either the highest or the lowest intensity depending on which is furthest from the vector median, which is only a good gradient measure for symmetric distributions.

The evaluation of (6) is also much less computationally expensive than the VRED and only requires ${ }^{N} C_{2}$ norms and the same number of comparisons. However, it suffers from the same sensitivity to noise as the classic morphological gradient. The MVRED overcame this problem by eliminating extrema vectors, as determined by their distance from the median. Likewise, the CMG can be made more robust by rejecting outlier vectors although, unless the vector median is explicitly calculated, it cannot use the same approach as the MVRED. Instead, a simple and effective technique is proposed that, having identified the two vectors that are furthest apart, removes all distances associated with them from the set of norms and then recalculates the maximum. This process can be repeated, successively removing pairs

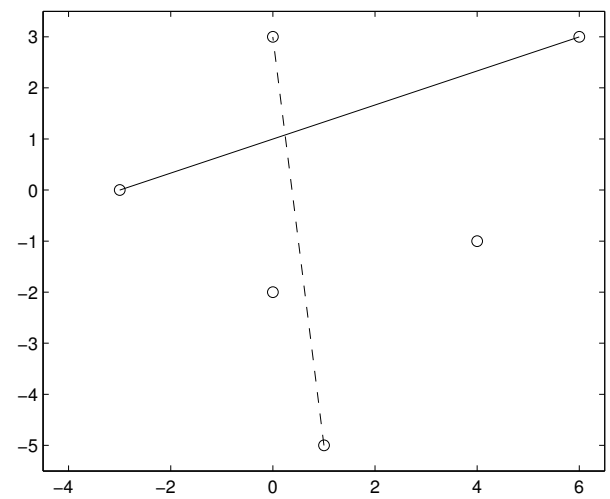

Fig. 1. Example colour morphological gradient operators using $L_{2}$ norm. The CMG of (6) and the RCMG of (7) for $n=1$ are the lengths of sold and dotted lines respectively.

of vectors and finding the new maximum. If $\left\{C M G_{\max _{n}}\right\}$ is the set of $n$ vector pairs eliminated, the robust colour morphological gradient (RCMG) can be defined by

$$
R C M G=\max _{i, j \in\{N\}-\left\{C M G_{\max _{n}}\right\}}\left\{\left\|\mathbf{X}^{(i)}-\mathbf{X}^{(j)}\right\|_{p}\right\} .
$$

For a set of $N$ vectors, $n$ cannot exceed $\frac{N-1}{2}-1$ if at least two vectors from the original set are to remain, although in practice lower values of $n$ are more appropriate. Figure 1 illustrates the RCMG for $n=1$ and, for noisy images, this process can be interpreted as follows. If any of the removed vectors is an outlier, such as that found with long-tailed noise, it will be successfully removed while if it comes from a relatively homogeneous population its removal will have little effect on the maximum norm as other, similar vectors will exist.

\section{EXPERIMENTAL RESULTS}

To evaluate the proposed techniques, they are applied to the $512 \times 512$ colour image Lenna. Figure 2 shows the original image and the results of the CMG and VRED using a $3 \times 3$ mask, the $L_{2}$ norm and the RGB colour space. For visualisation, the edge images are normalised to a $0-255$ range. It can be seen that although the CMG is computationally simpler its performance is very similar to that of the VRED both at edges and in the homogeneous regions. This result is interesting as its demonstrates that the explicit ordering used by the VRED and [9] is not a requisite for successful colour edge detection using morphological operators.

Figure 3 shows the response of the gradient operators in figure 2(b) and (c) for the portion of row 99 marked in figure 2(a). This location was chosen as it provides a crosssection through the boundary between Lenna's hat and the 


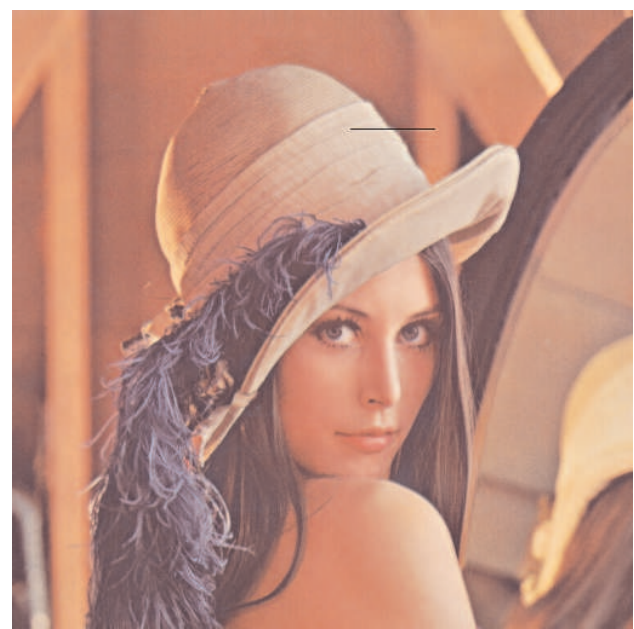

(a)

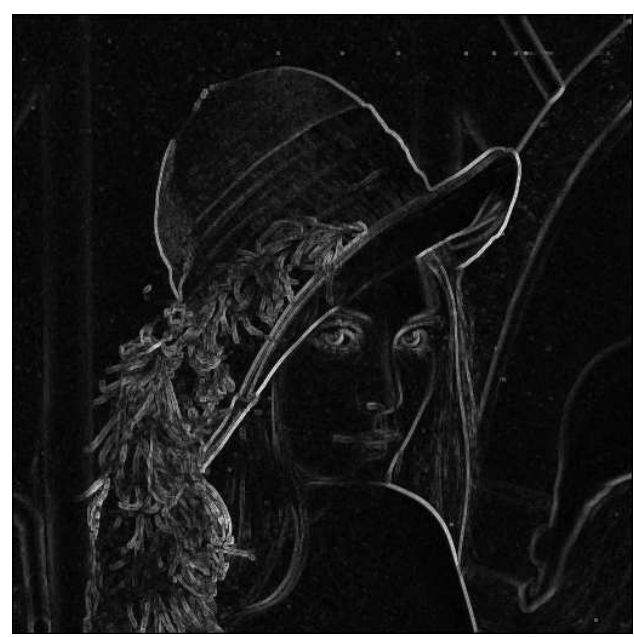

(b)

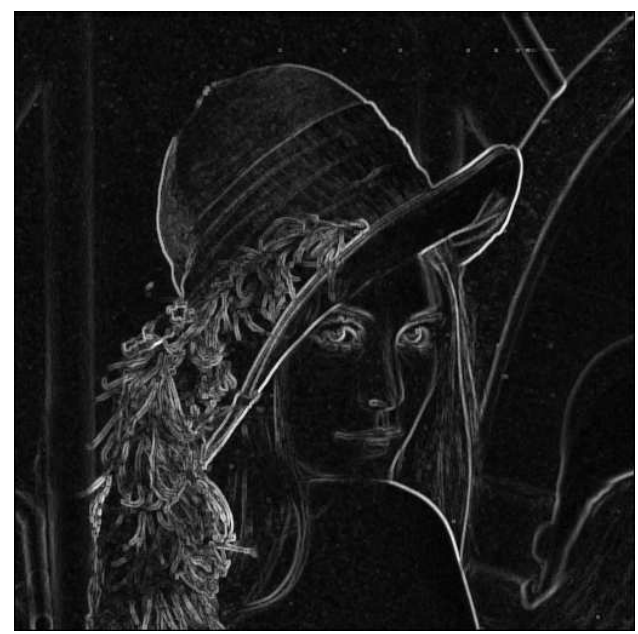

(c)

Fig. 2. Colour edge operator results: (a) original image showing position of cross-section used in figure 3 , (b) VRED result with $3 \times 3$ mask and (c) CMG for $3 \times 3$ mask.

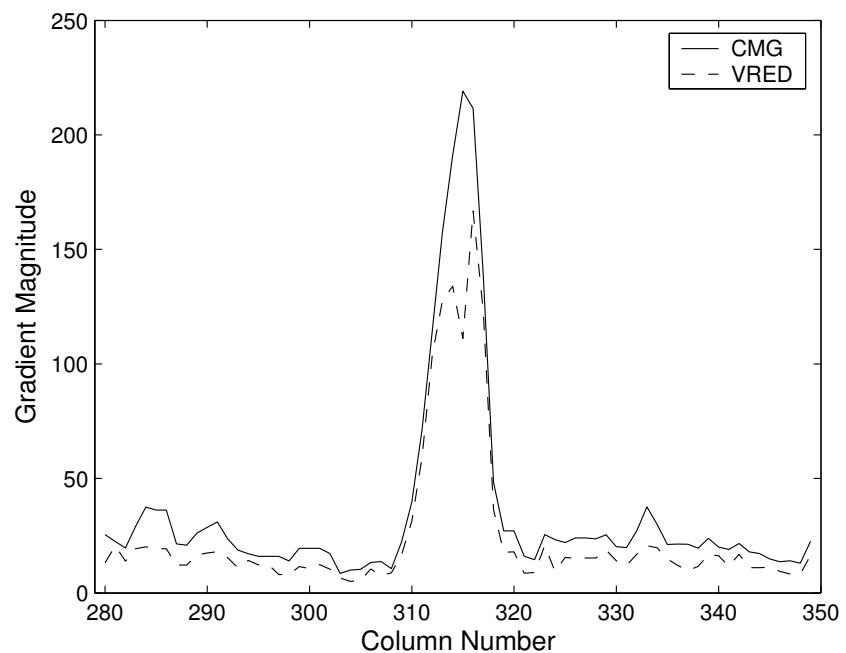

Fig. 3. CMG and VRED gradient magnitudes for row 99, columns 280-349 of Lenna, marked in figure 2(a) opposite.

background. In the hat and background regions both edge detectors provide a similar response, with the magnitude of the CMG above that of the VRED as would be expected from the theory. On the edge the VRED response shows a local minimum at the centre of the edge. This response, which was typical of those along this edge, shows that the one-sided difference between the vector median and vector extremum does not always estimate the true gradient. In contrast, the CMG response across the edge is well behaved and shows a peak at the desired position.

To test the sensitivity of the RCMG operator to noise, $15 \%$ of the pixels in Lenna were replaced by uniformly distributed random noise, see figure 4(a). For this level of noise it was found that neither the VRED nor the CMG produce a meaningful gradient image. With a mask size of $3 \times 3$ the RCMG result still suffers from noise, even when the maximum value of $n=3$ is used, see figure 4(b). This is initially somewhat surprising as only 3 vectors, giving 3 unique norms, remain. However, examination of the unprocessed image, where the high response is due to noise, reveals that they occur when 6 or more of the pixels in the $3 \times 3$ mask are noise points. When the mask size increases to $5 \times 5$ the results are improved, see figure 4 (b), with the only expense being a small reduction in the overall magnitude of the $\mathrm{CMG}$ response.

\section{CONCLUSIONS}

The CMG has been proposed as a morphological gradient operator for multivariate images. For a single channel input image, the CMG's output is identical to the classical morphological gradient, resulting in a filter that provides a good 
estimate of the true gradient in colour images. Its results are achieved without the computational expense of other nonlinear colour edge detectors, by virtue of the fact that it does not require the vectors to be ordered in order to evaluate the colour gradient.

As the response of the $\mathrm{CMG}$ is given by the maximum distance between any two points within the structuring element, it is sensitive to noise. To improve its robustness, outlier vector pairs can be eliminated before the maximum distance is determined, producing the RCMG. Again, by ranking pairs rather than individual vectors, an explicit vector ordering is not required. Results show that the RCMG performs well with noisy images providing the mask size is sufficient to contain the requisite number of noise-free pixels. Improving the performance of the CMG for short-tailed noise is an area of ongoing research.

\section{REFERENCES}

[1] A. Cumani, "Edge detection in multispectral images," Graphical Models and Image Processing, 53(1), pp. 40-51, 1991.

[2] G. Robinson, "Color edge detection," Optical Eng., 16(5), pp. 479-484, September 1977.

[3] M.A. Ruzon and C. Tomasi, "Color edge detection with the compass operator," in CVPR, 1999, pp. II: 160-166.

[4] M.A. Ruzon and C. Tomasi, "Edge, junction, and corner detection using color distributions," IEEE Trans. PAMI, 23(11), pp. 1281-1295, Nov. 2001.

[5] P.M. Djuric and J.K. Fwu, "On the detection of edges in vector images," IEEE Trans. IP, 6(11), pp. 15951601, 1997.

[6] J. Serra, Image Analysis and Mathematical Morphology, Academic Press, 1982.

[7] S. Beucher and C. Lantuéjoul, "Use of watersheds in contour detection," in Int. workshop on image processing, real-time edge and motion detection, Sept. 1979.

[8] J.S. Lee, R.M. Haralick, and L.G. Shapiro, "Morphologic edge detection," IEEE Trans. Rob. and Auto., vol. 3, pp. 142-156, 1987.

[9] M.C. d'Ornellas, "A multi-scale gradient approach for color-based morphological segmentation," in ICPR, 2000, pp. Vol-3 363-366.

[10] P.E. Trahanias and A.N. Venetsanopoulos, "Color edge detection using vector order statistics," IEEE Trans. IP, 2(2), pp. 259-264, 1993.

[11] P.E. Trahanias and A.N. Venetsanopoulos, "Vector order statistics operators as color edge detectors," IEEE Trans. SMC, 26(1), pp. 135-143, February 1996.

[12] J. Astola, P. Haavisto, and Y. Neuvo, "Vector median filters," Proc. IEEE, vol. 78, pp. 678-689, 1990.

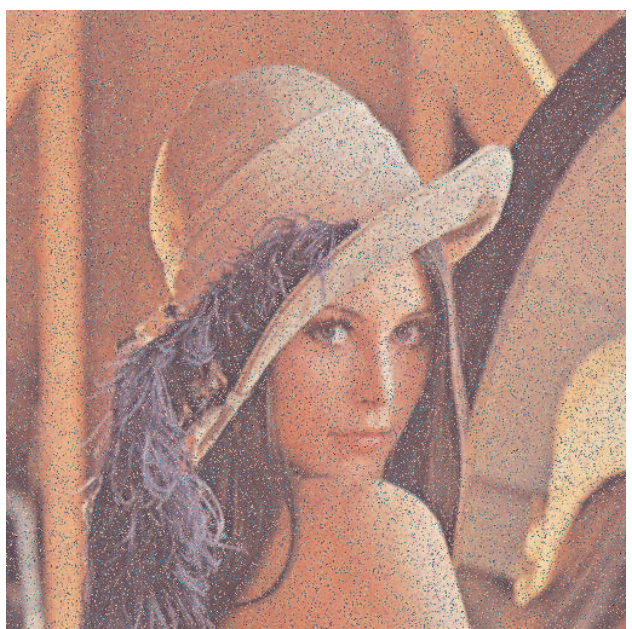

(a)

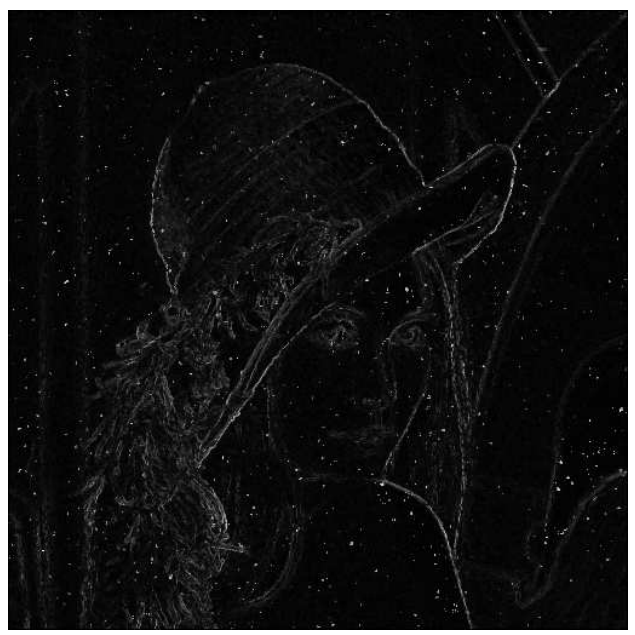

(b)

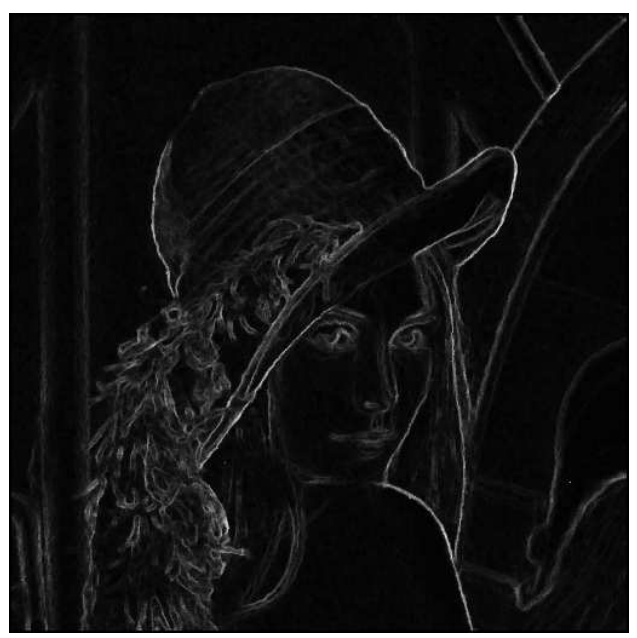

(c)

Fig. 4. RCMG results: (a) Lenna with $15 \%$ of pixels replaced with uniform random noise, (b) RCMG for $3 \times 3$ mask with $n=3$ and (c) RCMG for $5 \times 5$ mask with $n=9$. 